\title{
PALEOBIOGEOGRAFÍA DE CYRTINOPSIDAE (BRAQUIÓPO- DOS DELTHYRIDINA DEL SILÚRICO Y DEVÓNICO)
}

\author{
Jenaro L. GARCÍA-ALCALDE \\ Departamento de Geología (Paleontología), Universidad de Oviedo, C/ Jesús \\ Arias de Velasco s/n, 33005, Oviedo. c/e: jalcalde@uniovi.es.
}

García-Alcalde, J.L. 2005. Paleobiogeografía de Cyrtinopsidae (Braquiópodos Delthyridina del Silúrico y Devónico). [Paleobiogeography of the Cyrtinopsidae (Brachiopoda Delthyridina of the Silurian and Devonian]. Revista Española de Paleontología, 20 (2), 169-176. ISSN 0213-6937.

\begin{abstract}
The Family Cyrtinopsidae (Spiriferida, Suborder Delthyridina) comprises two subfamilies, Kozlowskiellininae and Cyrtinopsinae, representing successive steps of development. The oldest forms of both taxa originate during the Silurian, the Kozlowskiellininae in the Wenlock and the Cyrtinopsinae in the Pridoli. Their geographical origin was located in southern temperate latitudes, perhaps at the Bohemian end of Northern Gondwana. From here both family branches spread worldwide into the tropical belt. The Kozlowskiellininae took advantage of the meager extension of the so-called Rheic and Iapetus Oceans to reach Avalonia and Laurentia during the Late Silurian disappearing from both areas before the end of that period. However in Bohemia several new species arose from the main subfamily trunk during the Early Devonian. The youngest Kozlowskiellininae genus was Boucotiellina, from the Emsian of Southern China and North of Spain. The precise origin of Boucotiellina and the paleobiogeographic spreading of its species along Northern Gondwana are not yet known.

The Cyrtinopsinae exploited also the narrowing, even the disappearance, of oceanic barriers between Gondwana and northern paleocontinents to spread worldwide. The oldest genus of the subfamily, Megakozlowskiella, originated in Northeast Laurentia at the end of the Silurian and later reached Siberia westwards, and Australia and New Zealand eastwards, and vanished everywhere by the end of the Emsian. In turn, Cyrtinopsis produced several species during the Early and Middle Devonian in Northern Gondwana, Baltica and Siberia and disappeared by the Eifelian. Jehlanaria arose from Cyrtinopsis in Bohemia in Pragian times. In turn, Megakozlowskiella was the source of Plicocyrtina, with a few species known from the Pragian and Early Emsian of Alaska and Bohemia.
\end{abstract}

Key words: Paleobiogeography, Cyrtinopsidae, Silurian and Devonian.

\section{RESUMEN}

La Familia Cyrtinopsidae (espiriféridos del Suborden Delthyridina), está constituida por dos subfamilias, Kozlowskiellininae y Cyrtinopsinae que parecen representar sendos grados de desarrollo. Las formas más antiguas de ambos grupos aparecieron en el Silúrico, los Kozlowskiellininae en el Wenlock y los Cyrtinopsinae en el Pridoli. El origen geográfico de los Cyrtinopsidae se encuentra, posiblemente, en el extremo bohémico de Gondwana, desde donde las ramas de la familia se extendieron posteriormente a otros paleocontinentes en latitudes tropicales. Los Kozlowskiellininae aprovecharon la escasa extensión de los llamados océanos Reico y Japeto para colonizar, durante el Silúrico Superior, Avalonia y parte de Laurencia, extinguiéndose en dichas áreas antes de acabar el Silúrico. En su región de origen, sin embargo, el tronco principal de la subfamilia originó algunas nuevas especies a lo largo del Devónico Inferior. El taxón mas moderno del grupo parece ser Boucotiellina, del Emsiense de China Meridional y Norte de España. El origen concreto de Boucotiellina y las modalidades de dispersión de sus especies entre China Meridional y España son todavía desconocidas.

Los Cyrtinopsinae aprovecharon igualmente la reducción, incluso la desaparición, de barreras oceánicas entre Gondwana y los paleocontinentes septentrionales para extenderse prácticamente por todo el mundo. Megakozlowskiella, el género ancestral de la subfamilia, apareció en el nordeste de Laurencia hacia el final del Silúrico y alcanzó, más tarde, Siberia, por una parte, y Australia y Nueva Zelanda, por la otra, extinguiéndose a fines del Emsiense. A su vez, Cyrtinopsis produjo varias especies a lo largo del Devónico Inferior y Medio en el norte de Gondwana, Baltica y Siberia y desapareció en el curso del Eifeliense Inferior. Cyrtinopsis originó en el área 
bohémica una rama menor de vida efímera, Jehlanaria, durante el Praguiense. Megakozlowskiella sería, a su vez, la fuente de Plicocyrtina, género con especies conocidas en el Praguiense y Emsiense Inferior de Alaska y de Bohemia.

Palabras clave: Paleobiogeografía, Cyrtinopsidae, Silúrico y Devónico.

\section{INTRODUCCIÓN}

Determinar el origen y dispersión de las formas fósiles es un problema complejo que obliga a considerar simultáneamente factores paleogeográficos y paleoecológicos, y su expresión en secuencias temporales de taxones con relaciones de parentesco, evidenciadas en caracteres morfológicos compartidos y tendencias evolutivas particulares.
Algunas líneas filogenéticas están bien caracterizadas en el contexto referido, pero otras son más o menos enigmáticas y su conocimiento comporta numerosas lagunas.

Entre éstas últimas, se encuentra la Familia Cyrtinopsidae Wedekind, 1926 (Braquiópodos Espiriféridos Delthyridina). Dicho taxón comprendería dos subfamilias, Kozlowskiellininae Boucot, 1957a y Cyrtinopsinae Wedekind, 1926 (García-Alcalde, 2004), que se distribuyeron

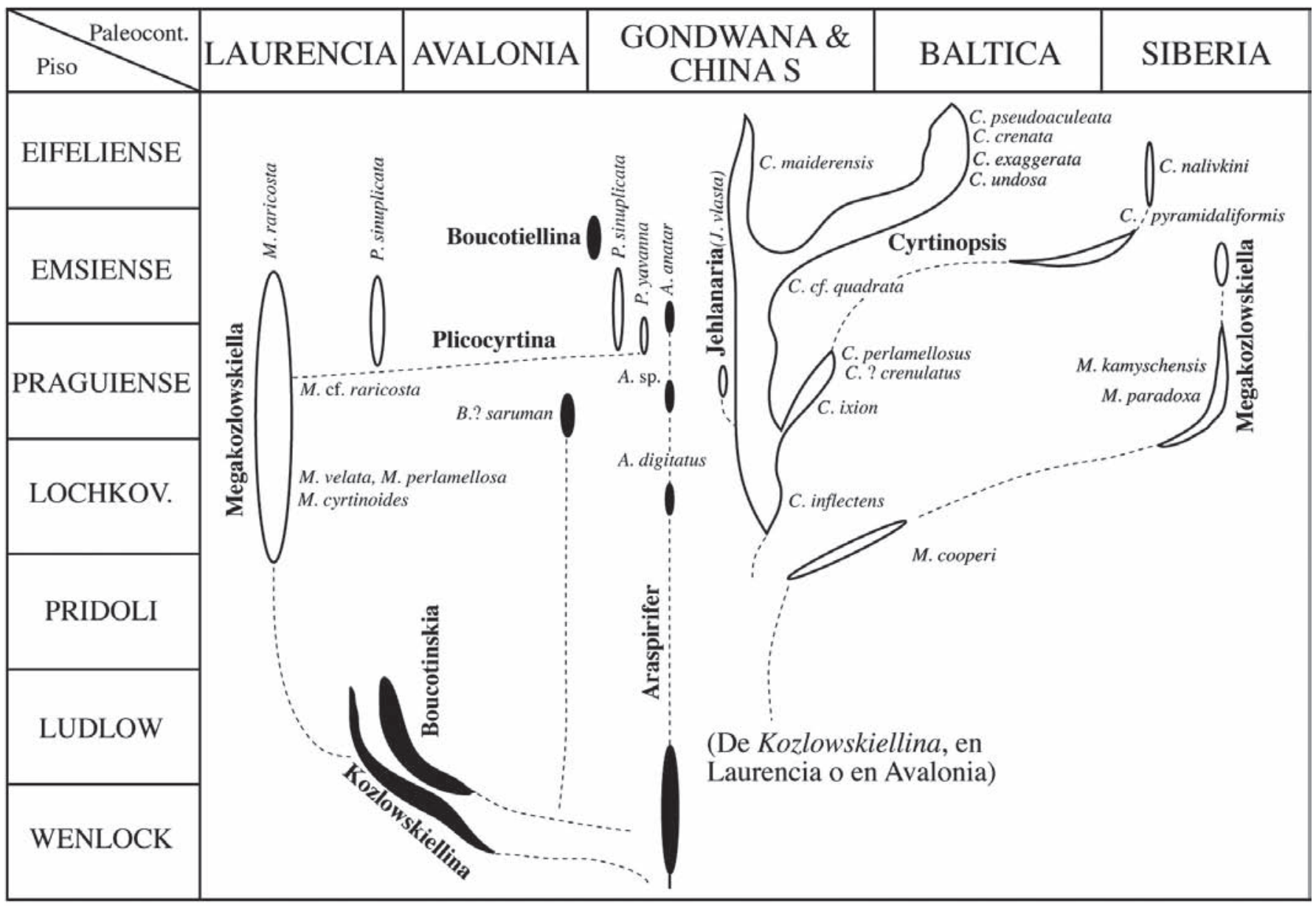

Taxones de la Subfamilia Cyrtinopsinae - Taxa of the Subfamily Cyrtinopsinae

Taxones de la Subfamilia Kozlowskiellininae - Taxa of the Subfamily Kozlowskiellininae

Figura 1. Distribución paleobiogeográfica de Cyrtinopsidae y posibles relaciones filogenéticas de los géneros de la familia. En el Devónico se incluyen las especies mejor conocidas. Abreviaturas, Paleocont.: Paleocontinentes; Lochkov.: Lochkoviense; China S: China Meridional.

Paleobiogeographical distribution of the Cyrtinopsidae genera and possible phylogenetic relationships among them. In the Devonian the best known species are included. Abbreviations, Paleocont.: Paleocontinents; Lochkov.: Lochkovian, China S.: Southern China. 
por casi todo el mundo durante el Silúrico y Devónico, y parecen representar sendos grados de desarrollo. El escalón basal estaría constituido por los Kozlowskiellininae, abundantes en el Silúrico Medio y Superior, con líneas prolongándose, eventualmente, hasta el Emsiense, mientras que el escalón superior abarcaría los géneros y especies de Cyrtinopsinae, de la parte final del Silúrico, pero abundantes sobre todo en el Devónico Inferior y Medio (Eifeliense) (Fig. 1).

\section{PALEOBIOGEOGRAFÍA DE LOS CYRTINOPSIDAE}

La Familia Cyrtinopsidae comprende un número reducido de géneros y especies, representadas habitualmente por pocos ejemplares en sus áreas de distribución. De acuerdo con García-Alcalde (2004), el taxón incluiría géneros caracterizados por la presencia de lamelas de crecimiento imbricantes, y apófisis cardinal y microornamentación microespinosa inexistentes o poco desarrollados.

Un detenido análisis bibliográfico y datos propios sobre el taxón más moderno de los Kozlowskiellininae, Воисоtiellina García-Alcalde, 2004, nos permite, sin embargo, ofrecer, con carácter preliminar, algunas pinceladas de la paleobiogeografía del grupo.

Las condiciones dinámicas terrestres, en el período de existencia de la familia y la ubicación geográfica de sus taxones a lo largo del tiempo, favorecen la idea de que la expansión del grupo se realizó, sobre todo, por dispersión a través de corredores o filtros, más que por fenómenos vicariantes. En el curso de los procesos migratorios, sin embargo, la génesis de formas nuevas requeriría la existencia de condiciones de aislamiento donde pudieron intervenir fenómenos de fragmentación de hábitats de carácter vicariante. El acceso de formas gondwánicas hacia la cuenca apalachense de Norteamérica, y eventualmente hacia Nevada, a partir del Emsiense, se produjo, con frecuencia, por la ruta migratoria "afro-apalachense" (Brice et al., 1997), sugerida por Oliver (1977) y Soto (1979). Pero en tiempos anteriores existió una ruta más directa, desde Meguma hacia la región de Gaspé, situada en el extremo septentrional de la referida cuenca apalachense (Boucot, 1960; Bouyx et al., 1997; Brice et al., 1997; García-Alcalde, 1999). Esta ruta sería un filtro bastante eficaz para las formas marinas debido a la existencia de importantes relieves desarrollados en el orógeno Acadiense y sólo permitiría el paso de unas pocas formas gondwánicas hacia el interior de Norteamérica y viceversa. Entre las que accederían a la cuenca Apalachense, se encontrarían, quizás, los antecesores kozlowskiellininos de Megakozlowskiella (Fig. 2 y 3). Un problema lo constituye, sin embargo, el que hasta el presente no se hayan descubierto fósiles de la Familia Cyrtinopsidae en Meguma (Brice et al., 1997).
Por su parte, Cyrtinopsis podría haber colonizado $\mathrm{Si}$ beria siguiendo la vía peri-báltica sugerida por GarcíaAlcalde (1999) extendiéndose luego hacia China Meridional (Fig. 3b).

El género de la familia con caracteres más primitivos parece ser Araspirifer Havlíček, 1987, acantonado en el territorio gondwánico de Bohemia desde el Wenlock hasta el Emsiense (Fig. 1). Pero hacia la misma época aparecieron, por lo menos, otras dos ramas distintas del tronco kozloswskiellinino, Boucotinskia Brunton \& Cocks, 1967 y Kozlowskiellina Boucot, 1958, con características igualmente muy generalizadas, cuyas especies se distribuyeron a lo largo de Avalonia y del Este de Norteamérica (Gran Bretaña, isla de Gotland, en Suecia, y estado de Nueva York) (Fig. 1 y 2) (Boucot, 1957a; Krans, 1969, 1973; Bassett \& Cocks, 1974). Todas estas formas, poseen placas dentales libres, bien desarrolladas, carecen de septo medio o lo tienen apenas indicado, y la apófisis cardinal falta o es muy simple. De acuerdo con la distribución geográfica que presentan, el centro de origen de todo el linaje cirtinópsido podría encontrarse tanto en Bohemia como en Avalonia pero, en cualquier caso, la dispersión posterior del grupo tiende a apoyar la idea de que, a partir del Wenlock, los antiguos brazos marinos Japeto y Reico (Fig. 2a-b), entre Laurencia y Báltica y entre Báltica y Gondwana, respectivamente, no representarían ya barreras ecológicas insalvables para los organismos bentónicos.

Del grupo cirtinópsido basal surgieron, a fines del $\mathrm{Si}$ lúrico, los primeros taxones de Cyrtinopsinae: Cyrtinopsis Scupin, 1896, en el saliente bohémico de Gondwana y Megakozlowskiella Boucot, 1957a, en el extremo septentrional de la cuenca Apalachense de Laurencia (Fig. 2b). Ambos géneros tienen septo medio ventral muy fuerte y muestran una tendencia clara a la pérdida de las placas dentales con formación de una cavidad espondiliar en Megakozlowskiella o de un verdadero espondilio, perforado por el septo medio, en Cyrtinopsis (Krans, 1971). La desaparición o reducción de las barreras oceánicas entre Gondwana y los paleocontinentes septentrionales durante el Devónico Inferior (Fig. 3a-b), permitió también la amplia dispersión de ambas formas. Megakozlowskiella se expandió por Laurencia durante el Devónico Inferior, alcanzando ya, en el Lochkoviense, Siberia, Australia y Nueva Zelanda (Gill, 1952; Krans, 1969, 1971, 1973; Boucot, 1957a; Lenz \& Johnson, 1985; Talent et al, 2001) (Fig. 1 y 3).

Un problema importante lo plantea la especie Kozlowskiellina baldisi Levy \& Nullo, 1972, del Devónico Inferior (Praguiense?-Emsiense Inferior?) de la Formación Talacasto, en Argentina. La figuración original de la especie muestra que pertenece, casi con toda seguridad, al género Megakozlowskiella. Sin embargo, ni la especie ni el género se citan entre las formas propias de la Fm. Talacasto en las recopilaciones recientes de Herrera (1993, 1995). También resulta sorprendente que un elemento propio de las regiones peri-ecuatoriales de casi todo el mun- 

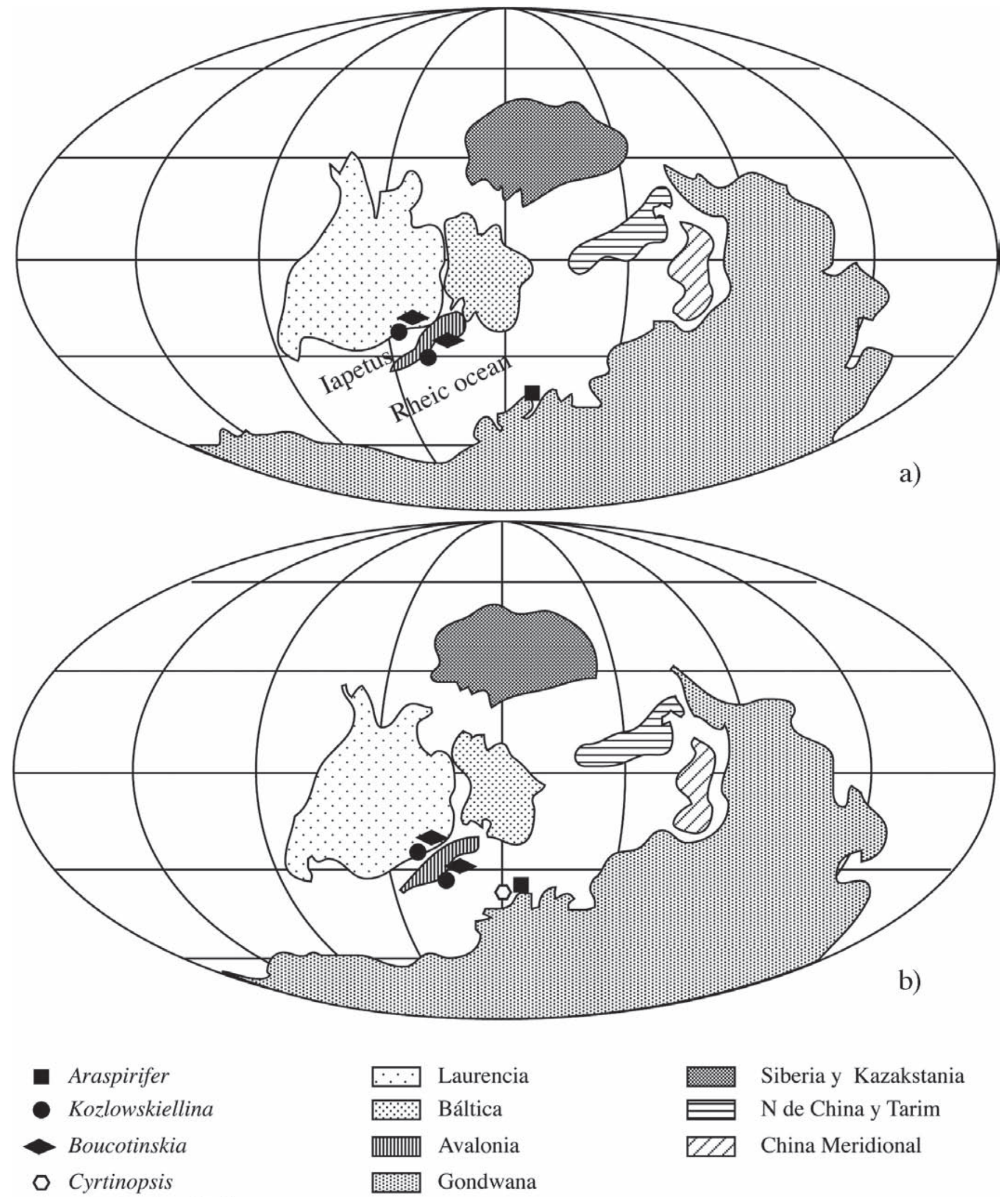

Laurencia

Báltica

Avalonia

Gondwana

$\Delta$ Megakozlowskiella

Figura 2. Paleobiogeografía de los géneros de la Familia Cyrtinopsidae (mapas paleogeográficos basados en Scotese \& McKerrow, 1990): a, Mediados del Silúrico (Wenlock). b, Finales del Silúrico (Ludlow-Prídoli).

Paleobiogeography of the genera of Cyrtinopsidae (paleogeographic maps based in Scotese \& McKerrow, 1990): a, Mid Silurian (Wenlock). b, End of Silurian (Ludlow-Pridoli). 
do, colonizase durante el Emsiense el ámbito facial malvino-africano, situado probablemente muy próximo al polo austral de la época.

Cyrtinopsis se encuentra durante el Lochkoviense, sólo en Gondwana septentrional (Fig. 1, 2b y 3a). Pero, en el curso del Praguiense, llegó a alcanzar, igual que Megakozlowskiella, incluso Siberia (Salair y Altai; Gratsianova et al., 1960; Kulkov, 1963; Rzhonsnitskaya, 1952; Gratsianova, 1967; Brice, 2000) (Fig. 1 y 3b). Megakozlowskiella se extinguió por todas partes hacia el Emsiense, pero Cyrtinopsis continuó su existencia hasta el Devónico Medio, mostrando una amplia distribución y elevada diversidad durante el Eifeliense (con varias especies en Báltica, Gondwana, China Meridional y, posiblemente también, en Siberia; Rzhonsnitskaya, 1952; Boucot, 1957b; Drot, 1964; Struve, 1965; Bublichenko, 1974; Su, 1976) (Fig. 1).

Una importante ramificación de Megakozlowskiella la representa Plicocyrtina Havlíček, 1956, con seno ventral costado y espondilio incipiente, conocido del Praguiense al Emsiense Inferior del Yukon, en Alaska y Bohemia (Havlíček, 1956; Lenz, 1972; Perry, 1984; Havlíček \& Vaněk, 1998) (Fig. 1). El supuesto origen de Plicocyrtina y su distribución parecen abonar la idea de que las especies del género recolonizarían el norte de Gondwana, procedentes de Laurencia (Fig. 3b). Por su parte, Cyrtinopsis originaría Jehlanaria Havlíček \& Vaněk, 1998 (conocido sólo por su especie-tipo, J. vlasta Havlíček \& Vaněk, 1998, del Praguiense de Bohemia) (Fig. 1 y 3b), con espondilio no perforado por el septo medio.

La idea de Talent et al. (2001) de que Cyrtinopsis evolucionaría de Plicocyrtina no parece bien substanciada, ni por las edades ni por las características morfológicas de ambos, y arrancaría de la determinación como Plicocyrtina de formas australianas y neozelandesas del Lochkoviense superior y Praguiense (Cyrtinopsis cooperi Gill, 1952). Dichas formas, se alejan de Plicocyrtina en la ausencia del pliegue medio sinal que da nombre al taxón. La morfología del interior de la valva ventral y el aspecto externo de la especie referida, son, más bien, propias de Megakozlowskiella, donde el mismo Talent (1963) y otros autores (Lenz \& Johnson, 1985) la incluyeron anteriormente, lo que tendería a apoyar la ramificación de Plicocyrtina a partir de Megakozlowskiella, que en este trabajo se acepta, sugerida hace tiempo por Boucot (1975).

Boucotiellina comprende, por ahora, sólo dos especies. La especie-tipo, B. ezquerrai (Verneuil, en Prado \& Verneuil, 1850), del Emsiense Superior de la Cordillera Cantábrica, en el norte de España, pertenece a una biocenosis propia de mares cálidos tropicales, no muy alejados del paleo-ecuador (García-Alcalde, 1995) donde proliferaron las faunas conchíferas con numerosas formas endémicas. Dicha biocenosis pertenecería a la Asociación Bentónica BA3 de Boucot (1975). Datos precisos sobre la paleoecología de la biocenosis fueron aportados por Alvarez \& Brime $(1982,1990)$ y Alvarez (1990). La especie china, $B$. acuta (Su, 1976), del Emsiense, por su parte, habitó también el área tropical, pero desconocemos los detalles de su paleoecología.

Boucotiellina plantea un problema especial dentro de la Familia Cyrtinopsidae. El taxón posee la morfología interna de Kozlowskiellina (donde fueron incluidas anteriormente sus especies por diferentes autores; Vandercammen \& Krans, 1964; Su, 1976), con placas dentales libres, bien desarrolladas, un menudo septo ventral y proceso cardinal bilobulado, muy simple (Fig. 4). Sin embargo, el género muestra rasgos originales como costillas, seno y pliegue medio dorsal muy agudos así como microornamentación microespinosa (Fig. 5). Pero si el grupo ancestral de Воисоtiellina hubiera sido Kozlowskiellina, existiría un largo período intermedio, de más de 15 millones de años (desde el Silúrico Superior al Emsiense), sin conexiones conocidas entre ambos taxones (Fig. 1). Los demás géneros de la Subfamilia Kozlowskiellininae, con representación en el Devónico, Araspirifer y Boucotinskia, difieren de Boucotiellina en importantes aspectos, tales como la posesión de cubierta deltidial, carencia de septo medio ventral, presencia de interárea ventral diferenciada, apófisis cardinal generalmente inexistente y falta de microornamentación microespinosa, por lo que no parecen candidatos plausibles como antecesores de éste. Por otro lado, tampoco se conocen formas de Boucotiellina a lo largo del área gondwánica, entre el norte del paleo-continente (Península Ibérica) y China Meridional, que pudiesen enlazar los extremos de la aparente distribución del género (Fig. 3b).

Los aspectos señalados llevan a concluir que aunque Boucotiellina podría ser el brote más moderno de la rama kozlowskiellinina de los Cyrtinopsidae, por el momento se desconoce su origen y la modalidad de dispersión paleobiogeográfica.

\section{AGRADECIMIENTOS}

Este trabajo se ha desarrollado en el marco del Proyecto "Origen de la Paleontología Asturiana. La Fauna del área de Ferroñes", de la Universidad de Oviedo y del IGCP 499, "Devonian land-sea interaction: evolution of ecosystems and climate". Todo mi agradecimiento a los revisores del artículo, Drs. Miguel V. Pardo Alonso y Peter Carls, por su importante contribución a la mejora formal del texto.

\section{BIBLIOGRAFÍA}

Alvarez, F. 1990. Devonian Athyrid brachiopods from the Cantabrian Zone (NW Spain). Biostratigraphie du Paléozoique, 11, 1-311.

Alvarez, F. \& Brime, C. 1982. Aportaciones al conocimiento de las condiciones de formación de algunos depósitos fosilíferos del Devónico Cantábrico. Trabajos de Geología de la Universidad de Oviedo, 12, 153-157. 

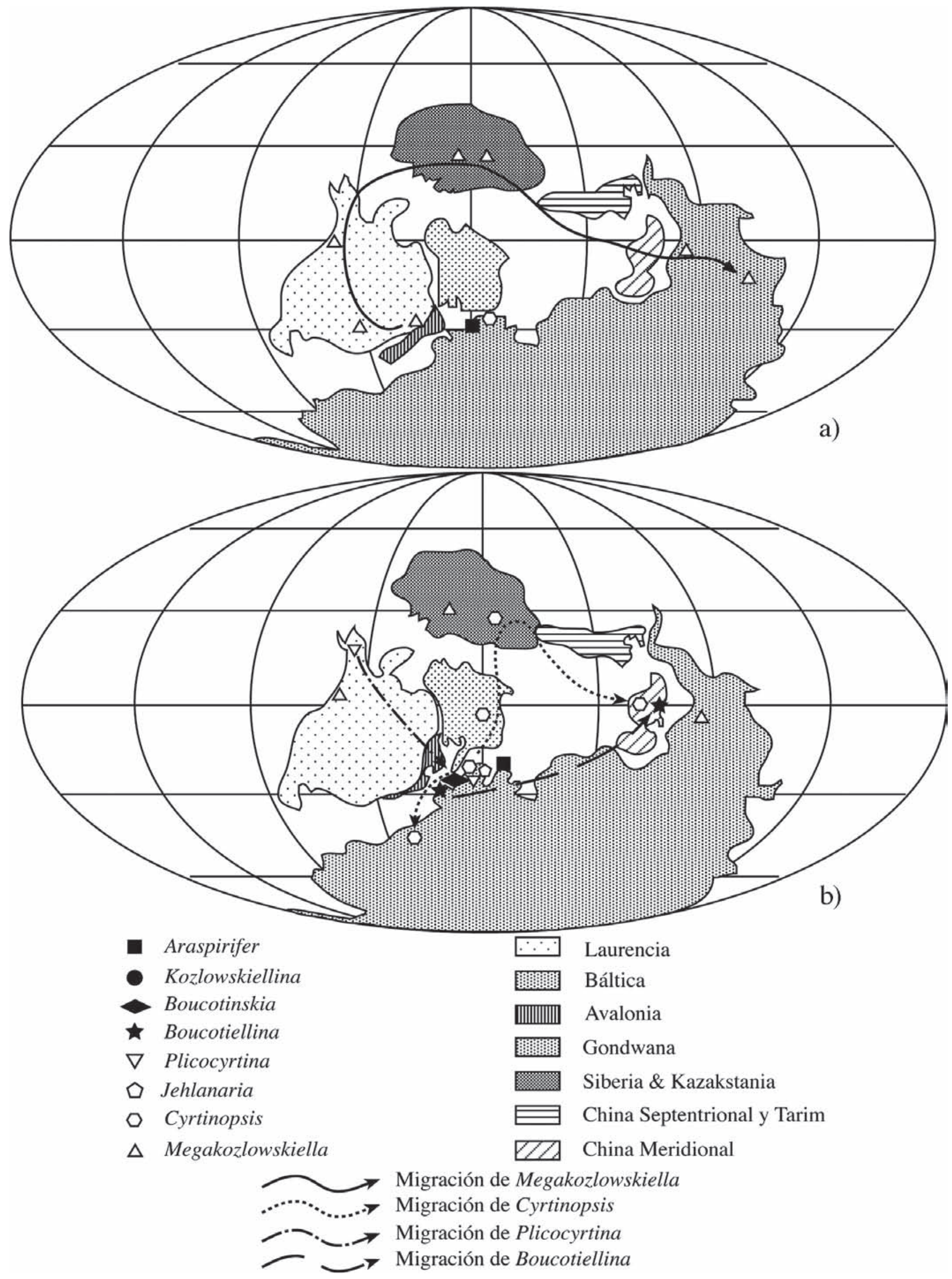


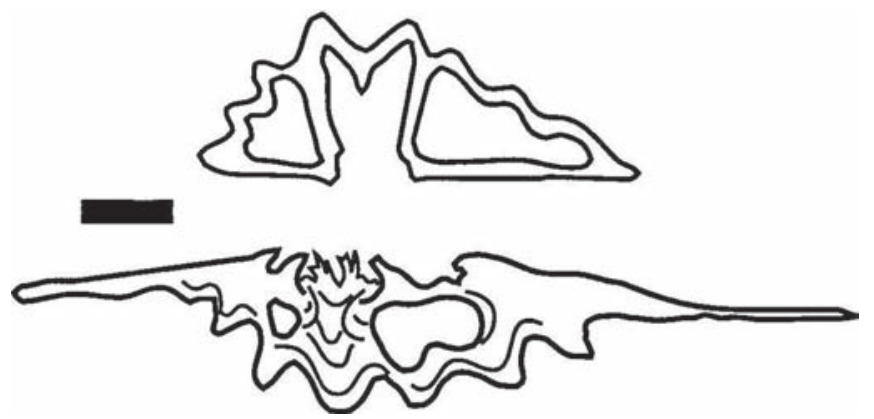

Figura 4. Sección transversal de un ejemplar de Boucotiellina ezquerrai (Verneuil, in Prado \& Verneuil, 1850), a 1,4 $\mathrm{mm}$ del ápice ventral, mostrando las placas dentales bien desarrolladas, el pequeño septo medio ventral y la apófisis cardinal rudimentaria. Barra horizontal: 1 $\mathrm{mm}$ (reproducido de García-Alcalde, 2004, con autorización del Institut royal des Sciences Naturelles de Belgique).

Serial transversal section of a specimen of Boucotiellina ezquerrai (Verneuil, in Prado \& Verneuil, 1850), to $1.4 \mathrm{~mm}$ from the ventral apex, showing the well developed dental plates, the small ventral median septum and the very simple cardinal process. Horizontal bar: $1 \mathrm{~mm}$ (reproduced from García-Alcalde, 2004, authorized by the Institut royal des Sciences Naturelles de Belgique).

Alvarez, F. \& Brime, C. 1990. Reencuentro con los "fósiles de Sabero". Comunicaciones de la Reunión de Tafonomía y Fosilización, Madrid, 13-22.

Bassett, M.G. \& Cocks, L.R.M. 1974. A review of Silurian brachiopods from Gotland. Fossils and Strata, 3, 1-56.

Boucot, A.J. 1957a. Revision of some Silurian and early Devonian spiriferid genera and erection of Kozlowskiellinae, new subfamily. Senckenbergiana lethaea, 38, 311-334.

Boucot, A.J. 1957b. A Devonian brachiopod, Cyrtinopsis, redescribed. Senckenbergiana lethaea, 38, 37-48.

Boucot, A.J. 1958. Kozlowskiellina, new name for Kozlowskiella Boucot, 1957. Journal of Paleontology, 32, 1031.

Boucot, A.J. 1960. Implication of Rhenish Lower Devonian brachiopods from Nova Scotia. 21 Congrès Géologique International, 12, 129-137.

Boucot, A.J. 1975. Evolution and extinction rate controls. Developments in Palaeontology and Stratigraphy, $\mathbf{1}$, Elsevier, 1-427.

Bouyx, E., Blaise, J., Brice, D., Dégardin, J.M., Goujet,, D., Gourvennec, R., Le Menn,, J., Lardeux, H., Morzadec, P. \& Paris, F. 1997. Biostratigraphie et paléobiogéographie du Siluro-Dévonien de la zone de Meguma (Nouvelle
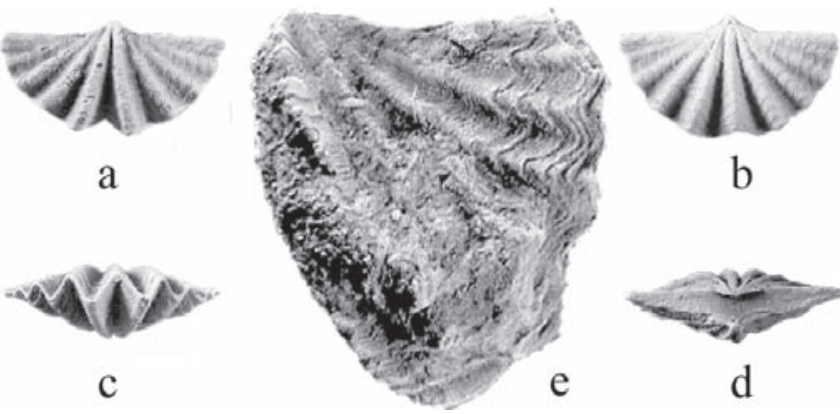

b

Figura 5. Boucotiellina ezquerrai (Verneuil, in Prado \& Verneuil, 1850), DPO 34348. a-d, Vistas ventral, dorsal, anterior y posterior, mostrando costillas y espacios intercostales muy agudos, x2. e, Valva ventral fragmentaria, DPO 34499 mostrando la micro-ornamentación micro-espinosa, $x 4$.

Boucotiellina ezquerrai (Verneuil, in Prado \& Verneuil, 1850), DPO 34348. a-d, Ventral, dorsal, anterior, and posterior views, showing the sharp angular ribs and similar interspaces, $x 2 . \boldsymbol{e}$, fragmentary ventral valve, DPO 34499, showing the microspinose micro-ornamentation. $x 4$.

Écosse, Canada). Canadian Journal of Earth Sciences, 34, 1295-1309.

Brice, D. 2000. Brachiopodes du Silurien supérieur et du Dévonien inférieur (Praguien probable) de Khemis n'Ga (region de Safi), Maroc. Travaux Institut Sciences Rabat, Séries Géologie \& Géographie Physique, 20, 11-24.

Brice, D., Gourvennec, R. \& Bouyx, E. 1997. Les brachiopodes dévoniens de la Zone de Meguma (Nouvelle-Ecosse, Canada) et les relations entre le Royaume du Vieux Monde et la province Appalachienne. Geobios, M.S., 20, 75-78.

Brunton, C.H.C. \& Cocks, L.R.M. 1967. Proposed suppression under the plenary power of three species names of brachiopods erected by Linnaeus (1758). Z.N. (S.) 1810. Bulletin of Zoological Nomenclature, 24, 297-300.

Bublichenko, N.L. 1974. Brakhiopode srednego i verchnego Devona Rudnogo Altaya. Izdatel'stvo Nauka Kazakhskoi SSR. Alma-Ata, 1-169.

Drot, J. 1964. Rhynchonelloidea et spiriferoidea Siluro-Dévoniens du Maroc pré-saharien. Notes et Mémoires du Service Géologique du Maroc, 178, 1-286.

García-Alcalde, J.L. 1995. L'évolution paléobiogéographique pré-varisque de la Zone Cantabrique septentrionale (Espagne). Revista Española de Paleontología, 10, 9-29.

Figura 3. Paleobiogeografía de los géneros de la Familia Cyrtinopsidae (mapas paleogeográficos basados en Scotese \& McKerrow, 1990). a, Comienzos del Devónico (Lochkoviense); b, Praguiense-Eifeliense.

Paleobiogeography of the genera of Cyrtinopsidae (paleogeographic maps based in Scotese \& McKerrow, 1990. a, Earliest Devonian (Lochkovian). b, Pragian to Eifelian. 
García-Alcalde, J.L. 1999. Paleogeography of Spanish lower Devonian Rhynchonellids. Bollettino della Società $\mathrm{Pa}$ leontologica Italiana, 37, 543-545.

García-Alcalde, J.L. 2004. Lower Devonian Delthyridoidea (Brachiopoda, Delthyridinae) of the Cantabrian Mountains (N Spain). Bulletin de l'Institut royal des Sciences Naturelles de Belgique, 74, 9-38.

Gill, E.D. 1952. Palaeogeography of the Australian-New Zealand region in Lower Devonian time. Transactions and Proceedings of the Royal Society of New Zealand, 80, 171-185.

Gratsianova, R.T., Zinchenko, V.G. \& Kulkov, N.P. 1960. Tip Brachiopoda. Plechenogie.V.Kh.Biostratrigrafiya Paleozoi Sayno-Altaiskoi Gornoi oblasti.T.II. Izdatel'stvo Novosibirsk, Sniiggims, 422-480.

Gratsianova, R.T., 1967. Brakhiopody i stratigrafiya Nichnego Devona Gornogo Altaya. Izdatel'stvo "Nauka”, Sibirskoe otdelenie. Instituti Geologii i Geofiziki, 1-160.

Havlíček, V. 1956. The brachiopods of the Branik and Hlubocepy Limestones in the immediate vicinity of Prague. Sbornik Ústredniho ústavu geologického, 22, 1-131.

Havlíček, V. 1987. New genera of Silurian brachiopods. Věstnik Ústředniho ústavu geologického, 62, 239-244.

Havlíček, V. \& Vaněk, J. 1998. Pragian brachiopods, trilobites, and principal biofacies in the Prague Basin (Lower Devonian, Bohemia). Sbornik Geologickych ved, Paleontologie, 34, 27-109.

Herrera, Z.A. 1993. Nuevas precisiones sobre la edad de la Formación Talacasto (Precordillera Argentina) en base a su fauna de braquiópodos. Actas XII Congreso Geológico Argentino y II Congreso de Exploración de Hidrocarburos, 2, 289-295.

Herrera, Z.A. 1995. The Lower Devonian Chonetoidean brachiopods from the Argentine Precordillera. Documents $d u$ Laboratoire Géologique de Lyon, 136, 101-147.

Krans, T.F. 1969. Morphological observations on Kozlowskiellina (Spiriferida). Leidse Geologische Mededelingen, 44, 265-307.

Krans, T.F. 1971. The relation between the genera Cyrtinopsis Scupin, 1896 and Kozlowskiellina Boucot, 1957. Leidse Geologische Mededelingen, 4, 99-113.

Krans, T.F. 1973. On the phylogenetic position of the genus Boucotinskia Brunton and Cocks, 1967 (Spiriferida). Leidse Geologische Mededelingen, 49, 277-283.

Kulkov, N.P. 1963. Brakhiopody solovikhinskikh sloev'stnichnego Devona Gornogo Altaya. Izdatel'stvo “Nauka”, Sibirskoe otdelenie, Akademii Nauk SSSR, $1-131$

Lenz, A.C. 1972. Plicocyrtina and Plicoplasia (Brachiopoda) from the Lower Devonian of the northern Canadian Cordillera. Journal of Paleontology, 46, 99-103.

Lenz, A.C. \& Johnson, B.D. 1985. Brachiopods of the Garra Formation (Lower Devonian), Wellington Area, New South Wales, Australia: Rhynchonellida, Spiriferida, Terebratulida. Palaeontographica, A, 188, 71-104.
Levy, R. \& Nullo, F.E. 1972. Spiriféridos Devónicos de Argentina 1. Ambocoelia y Kozlowskiellina. Ameghiniana, 9, 45-58.

Oliver, W.A. 1977. Biogeography of late Silurian and Devonian rugose corals. Palaeogeography, Palaeoclimatology, Palaeoecology, 22, 85-135.

Perry, D.G. 1984. Brachiopoda and biostratigraphy of the Silurian-Devonian Delorme Formation in the District of Mackenzie, the Yukon. Life Science Contributions, Royal Ontario Museum, 138, 1-243.

Prado, C. de \& Verneuil, E. de 1850. Note géologique sur les terrains de Sabero et de ses environs dans les montagnes de Léon (Espagne) (C. de Prado), suivie d'une description des fossiles de ces terrains (E. de Verneuil). Bulletin Société Géologique de France, ser. 2, 7, 137-186.

Rzhonsnitskaya, M.A. 1952. Spiriferidy Devonskikh otlochenii Okkrani Kuznetskogo basseina. Trudy Vsecofznogo Nauchno-Issledovatelskogo Geologicheskogo Instituta (VSEGEI), 1-232.

Scupin, H., 1896. Versuch einer Classification der Gattung Spirifer. Neues Jahrbuch Mineralogie, Geologie und Paläontologie, 2, 239-248.

Soto, F. 1979. Considérations paléobiogéographiques sur les Streptelasmatina (Coelenterata, Rugosa) solitaires du Dévonien des Monts Cantabriques (NW de l'Espagne). Geobios, 12, 399-409.

Struve, W. 1965. Beiträge zur Kenntnis devonischer Brachiopoden, 13: Zur Morphologie, Biochronologie und Phylogenie der mitteleuropäisch-nordafrikanischen CyrtinopsisArten (Spiriferacea). In: Das Mitteldevon des Rheinischen Schiefergebirges. Ein Symposium, Fortschritte in der Geologie von Rheinland und Westfalen, 9, 7-50.

$\mathrm{Su}$ Yang-Zheng 1976. Cambrian-Devonian Brachiopoda (en chino). Paleontological Atlas of North China, Nei Mongol volume, 1 (Geological Bureau Nei Mongol Autonomous Region \& Northeast Institute Geological Sciences), 159227.

Talent, J.A. 1963. The Devonian of the Mitchell and Wentworth rivers. Geological Survey Victoria, 24, 1-117.

Talent, J.A., Gratsianova, R.T. \& Yolkin, E.A. 2001. Latest Silurian (Pridoli) to middle Devonian (Givetian) of the Asio-Australia hemisphere: rationalization of brachiopod taxa and faunal lists; stratigraphic correlation chart. Courier Forschungsinstitut Senckenberg, CFS, 236, 1-221.

Vandercammen, A. \& Krans, T.F. 1974. Révision de quelques types de Spiriferidae d'Espagne. Bulletin Institut royal Sciences naturelles Belgique, 40, 1-40.

Wedekind, R. 1926. Die Devonische Formation. In: Salomon, W.A. Grundzüge der Geologie, 2, Erdgeschichte, 194-226.

Manuscrito recibido: 10 de Octubre, 2004 Manuscrito aceptado: 1 de Octubre, 2005 